\title{
ALERTNESS, VISUAL COMFORT, SUBJECTIVE PREFERENCE AND TASK PERFORMANCE ASSESSMENT UNDER THREE DIFFERENT LIGHT'S COLOUR TEMPERATURE AMONG OFFICE WORKERS
}

\author{
SHAMSUL, B.M. T. ${ }^{\mathrm{a} 1}$, NUR SAJIDAH, S. ${ }^{\mathrm{b} 1}$, ASHOK, S. ${ }^{\mathrm{c} 2}$ \\ ${ }^{1}$ Department of Environmental and Occupational Health, Faculty of Medicine and Health Sciences, \\ Universiti Putra Malaysia Selangor \\ ${ }^{2}$ Product Quality \& Reliability Engineering MIMOS Berhad, Kuala Lumpur, Malaysia

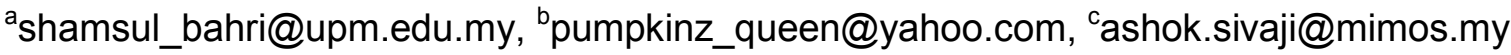

KEYWORDS: Lighting; correlated colour temperatures; visual comfort; alertness level.

\begin{abstract}
Different CCT provided by different lighting are important in affecting human beings psychologically and physiologically, as well as visual and non-visual processes. This study aim in finding out the effects of warm white (WW) light $(\mathrm{CCT}=2700 \mathrm{~K})$, cool white $(\mathrm{CW})$ light $(\mathrm{CCT}=4000 \mathrm{~K})$ and artificial Daylight $(\mathrm{CCT}=6200 \mathrm{~K})$ on worker's alertness, visual comfort level, preferences and task performance. A repeated measure experimental study was conducted among 46 office workers under controlled environment. Alertness levels were measured by Karolinska Sleepiness Scale. Office Lighting Survey was used to evaluate visual comfort and preference. Typing speed and accuracy also were tested. Paired Sample T-Test was used and the results showed that there was significant reduction of subjective alertness level in WW light $(p=0.001)$. In term of typing performances, subjects performed significantly better in term of typing speed under CW light and Daylight than WW light. Less typing errors were made under DL, followed by CW light. Meanwhile WW light lead to high typing errors. Besides, CW light is the most preferred whereas subjects feel most comfortable under Daylight. Subjects indicated that they can perform longer works in this coloured-lit environment. The $\mathrm{CW}$ light and DL light were more beneficial for office setting for computer-based task.

\section{Introduction}

Good lighting is required for good visibility of the environment. Lighting should ensure the luminous environment that is human-friendly and appropriate for the visual task performed. Various studies had been conducted in order to identify the effects of lighting condition towards human health, productivity, well-being and alertness level [1]. In regards of human perception, two of the most important characteristics of lights are illuminance and correlated colour temperature (CCT) [2]. Recently, studies had proven that different CCT provided by different lighting are important in affecting human psychologically and physiologically, as well as visual and non-visual processes [3]. The right selections of CCT in an office environment will benefits occupants in term of visual comfort and reduction of daytime sleepiness. This will increase of productivity and prevention of health effects associated with inappropriate light's CCT such as eye strain, or the effects towards emotion and human circadian rhythm [4]. The objective of this study is to identify the differences in term of alertness level, typing performance and subjective visual comfort and preferences among office workers when exposed under different light's CCT (WW 2700K, CW 4000K and DL $6200 \mathrm{~K})$.
\end{abstract}




\section{Methodology}

This experiment was performed in MIMOS UX Lab, MIMOS Berhad located at Bukit Jalil, Malaysia. With a dimension of $14 \mathrm{ft} \times 13 \mathrm{ft}$, the room was illuminated with 8 ceiling bulb Philips TLD 36W/827 (warm white, 2700K) for the first lighting condition, 8 ceiling bulb PHILIPS TL-D 36W/840 (Cool white, 4000K) for second lighting condition and 8 ceiling bulb PHILIPS TL-D $36 \mathrm{~W} / 865$ (Day Light, 6200K) for the third lighting condition. The environmental is controlled in terms of illuminance setting of 400 lux, temperature settings of $28^{\circ} \mathrm{C}$ and ergonomic sitting position. This controlled environmental factor is chosen based on the recommended indoor environmental condition of an office setting [5].
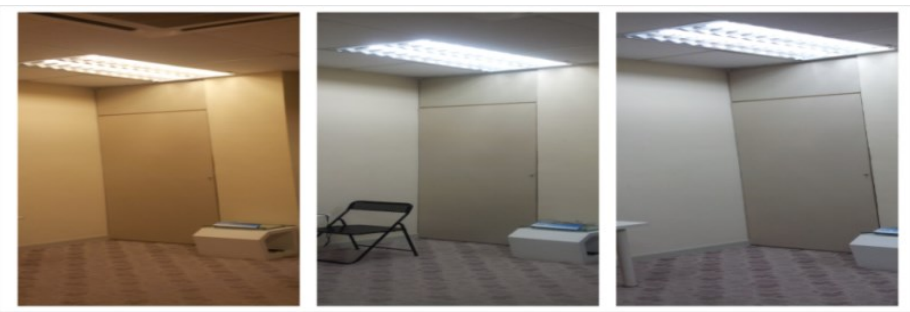

Figure 1: Light setting of the Usability Testing Laboratory in MIMOS Berhad (a) 2700K; (b) $4000 \mathrm{~K}(\mathrm{c}) 6200 \mathrm{~K}$.

\section{Alertness level, Visual comfort and subjective preferences}

In each experiment, the respondent's alertness was monitored using the established Karolinska Sleepiness Scale (KSS) [6]. KSS is a set of questionnaire for subjects to rate their actual alertness level on a 9-stage scale from being "extremely alert" $(=1)$ to "very sleepy, great effort to keep awake, fighting sleep" (=9). The higher the score of KSS indicate lower level of alertness. Modified Office Lighting Survey (OLS) is a questionnaire-based assessment method for occupant satisfaction regarding office lighting using a mix of general and artificial lighting-specific statements (7-point Likert scale) [8].

\section{Percentage of eye fixation}

Tobii $^{\circledR}$ T60 Eye Tracker was used to measure numbers of fixation on the computer screen with the aim of observing the impact of different light's color temperature in subject's performance by measuring the numbers of fixation on the screen while performing tasks. The number of fixation is a measure of total gazes (fixations) captured by the eye tracker from the start to the end of performing a specified task as reported in other studies [9]. For each lighting conditions, the number of fixations and the accuracy of performing the task were recorded.

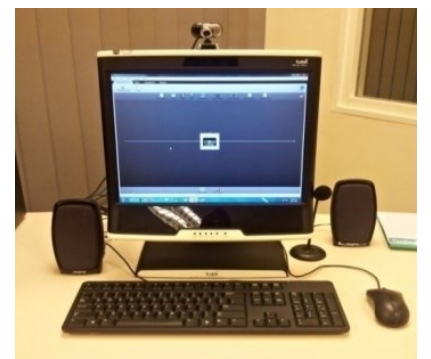

Figure 2: Tobii Eye Tracker T60.

\section{Typing Test}

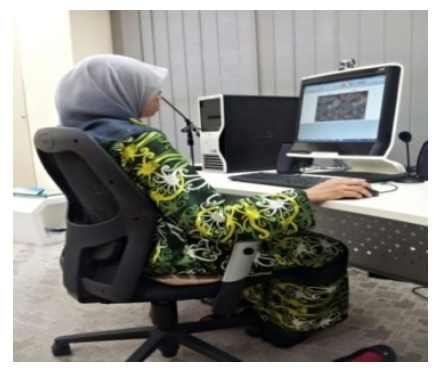

Figure 3: Eye tracker is measuring the numbers of eve fixation

To determine the optimum CCT for typing performance, subjects were required to type an article using Microsoft Word 2007 by referring to a printed article within the duration of 10 minutes. Three different articles (approximately 400 words) were prepared with similar level of difficulty. The automatic spelling and grammar checking were disabled as the aim of this study is to 
measure the accuracy of the subject's typing performance under various lighting conditions: typing speed (total numbers of words typed) and typing accuracy (percentage of typing errors).

\section{Result and Discussion \\ Alertness level}

Figure 4 shows the comparison of alertness level before and after the trial. There is increment of alertness level score of subjects under WW light (Mean difference, +0.6) towards the end of the experiment as compared to CW light and Daylight. The higher the scores, indicates that the lower the alertness level.

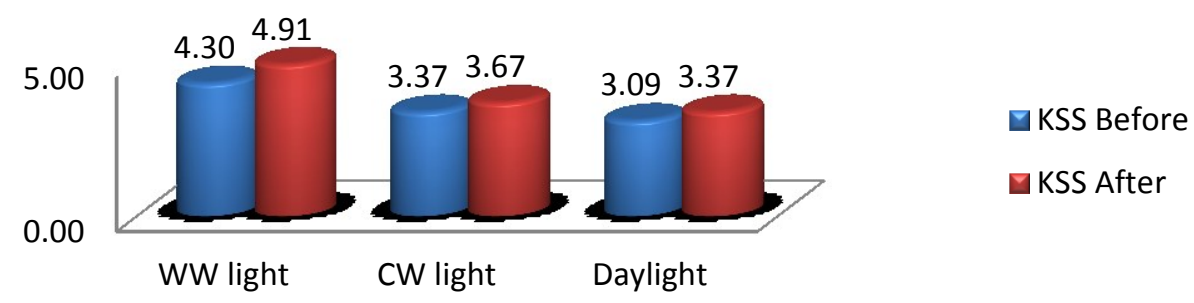

Figure 4: Comparison of alertness level before and after the trial

Statistical analysis shows that there was significant changes in alertness level of subjects when exposed under WW light (Paired sample T-test, $\mathrm{p}=0.001<0.05$ ) which means alertness level decreased towards the end of the experiment. In contrast, under CW light $(p=.056>0.05)$ and Daylight $(p=0.113, p>0.05)$ there are no significant changes observed. This is supported by previous research whereby Daylight is said to have increased the alertness level of subjects. High CCT was associated with better alertness [13] as studies showed that subjects feels less sleepy, tedious and undesirable under high levels of colour temperature $(5000 \mathrm{~K} \& 6000 \mathrm{~K})$ compared to condition with lower CCT of light (3000K \& 4000K) [14]. On the other hand, the result obtained is in contrast with most of previous studies conducted where higher CCT of lights is said to have effects in the feelings of increase alertness level among office workers [15].

\section{Visual comfort and subjective preferences}

Figure 5 shows that subjects were most comfortable under Daylight (25.36 points). The results of r-ANOVA show that there are significant differences between visual comfort levels of respondents when exposed under the three different CCT of lights' $F(1.80,81.06)=62.75, p=0.00$.

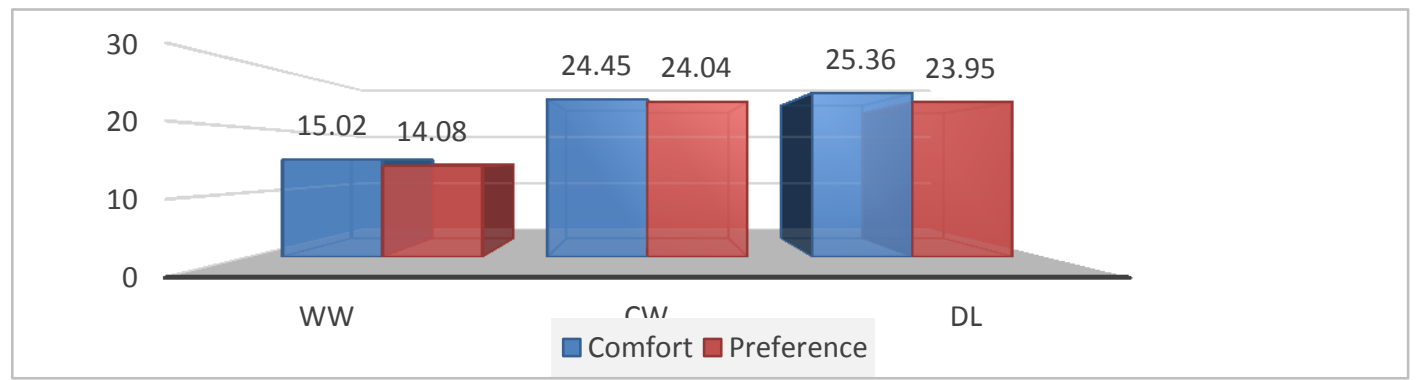

Figure 5: Comparison of visual comfort level and preferences under three different CCT of light (mean).

In terms of preference, it was found that subjects significantly preferred CW light over WW light ( $\mathrm{p}=0.000,<0.05)$. Besides that, $\mathrm{CW}$ light is also considered as comfortable light's CCT (24.45 points) as compared to WW (15.02 points) with the statistical analysis showing significant different ( $r$-ANOVA, $p=0.00, p<0.05$ ). Furthermore, Daylight (23.95 points) is also considered as preferable light's CCT as compared to WW (14.08 points) (r-ANOVA $\mathrm{p}=0.00,<0.05)$. Subjective preference on light's CCT varies between individual as reported by Miller (2007) [16] found that the 
preferences of lighting condition varies between people and even to an extent of the same individual during different times of a day. In contrast, another study suggested that there are no trends of individual preference in choice of light colour in an office environment [17].

\section{Percentage of eye fixation}

Figure 6 shows that Daylight have the highest average number of eye fixation $(75.26 \%)$ followed by WW light (72.65\%) and CW light (71.52\%). R-ANOVA analysis indicated that there are no significant different between the three different light's CCT and number of eye fixation $(p=0.486>0.05)$. This shows that different CCT of lights does not influence the numbers of eye fixation on the computer screen. Based on this study, the highest percentage of eye fixation by Daylight $(75.26 \%)$ might be due to the enough amount of lighting entering the pupil thus helping the eye tracker to record the fixations of subjects while conducting the entire visual tasks.

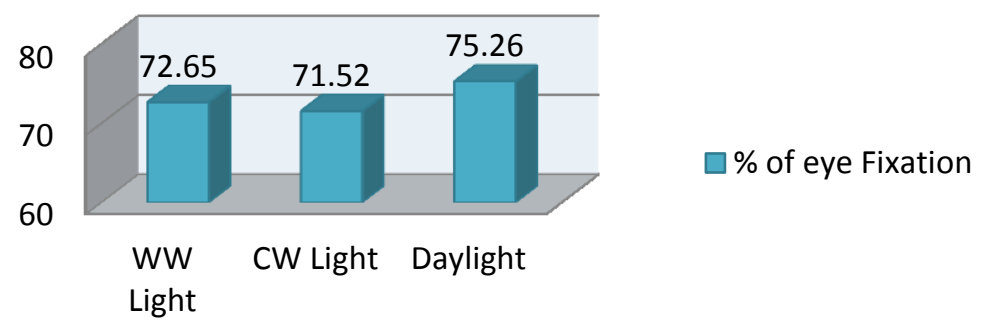

Figure 6: Comparison of percentage of eye fixation under different CCT of lights (mean).

\section{Typing Test}

Table 3 shows the comparison of mean score for typing speed under three different CCT of lights. For typing test, subjects working under the CW light responded with the highest typing speed of an average of $295.24 \pm 8.48$ words, followed by Daylight having $287.35 \pm 8.78$ words. Subjects under the WW light had the slowest typing speed of $269.11 \pm 9.68$ words. Further statistical analysis using r-ANOVA show that there are significant differences of typing speed among office workers when exposed under the three different CCT of lights $F(1.73,77.96)=20.045, p=0.00$.

Table 3: Comparison of mean score for typing speed under three different CCT of lights

\begin{tabular}{cccc}
\hline $\begin{array}{c}\text { Numbers of words typed under } \\
\text { lighting scenarios }\end{array}$ & Min. & Max. & Mean \pm SD. \\
\hline WW Light & 121 & 412 & $269.11 \pm 9.68$ \\
CW Light & 189 & 408 & $295.24 \pm 8.48$ \\
Daylight & 175 & 384 & $287.35 \pm 8.78$ \\
\hline
\end{tabular}

On the other hand, by comparing the mean of error percentage, CW light has the lowest mean $(2.85 \pm 0.33)$ which indicated that respondents work efficiently by conducting less numbers of errors under this lighting condition as compared to other CCT of lights. In contrast, WW light resulted in the highest mean of words typed $(3.65 \pm 0.79)$ which indicated that respondent performance in typing under this lighting condition is less efficient (high percentage of errors) as compared to other CCT of lights exposed. Further statistical analysis using r-ANOVA concluded that typing accuracy is not affected by the differences of lights' CCT. The results show that there are no significant differences of typing accuracy among office workers when exposed under the three different CCT of lights $F(1.662,160.226) \mathrm{p}=0.997, \mathrm{p}=0.361$ (Table 4). 
Table 4: Comparison of typing accuracy of subjects under different light's CCT.

\begin{tabular}{ccccc}
\hline $\begin{array}{c}\text { Numbers of wrong words typed } \\
\text { under lighting scenarios }\end{array}$ & Min. (\%) & Max. (\%) & \multicolumn{2}{c}{ Mean \pm SD. } \\
\hline WW Light & 0.67 & 37.50 & 3.65 & \pm 0.79 \\
CW Light & 0.36 & 13.56 & 2.86 & \pm 0.33 \\
Daylight & 1.06 & 12.04 & 3.22 & \pm 0.32 \\
\hline
\end{tabular}

Our results show the link between speed of typing and visual comfort. As subjects felt most comfortable under CW light, they typed significantly faster than WW light (least comfortable perceived). This finding is in line with previous study conducted among university students whereby CW lights is said to have significantly faster typing speed and work performance compared to WW light and Daylight $[14,20]$. In addition, another study also pointed out that WW light was less effective in information processing (looking at paper and reading) and task (typing) [21].

For typing accuracy, subjects committed the least amount of errors under CW light setting. This is because subject feels comfortable working under CW light which can be related with the subjects' level of alertness whereby $\mathrm{CW}$ light did not lead to any significant changes of alertness level among respondents. Therefore, error performed by subjects is more when they are exposed under WW light as the level of alertness reduced [20]. This finding is similar with previous study conducted among university students [21].

\section{Conclusion and Recommendation}

As a conclusion, subject working under the WW light recorded the highest reduction in level of alertness while CW light and Daylight resulted with no significant changes of alertness level in the end of experiment. In addition Daylight is the most comfortable CCT of light's while CW light is found to be the most preferred light's CCT. Meanwhile, numbers of eye fixation on computer screen is not affected by the difference of light's CCT. Hence, there is no recommendation for the optimal lighting that involves office workers using eye tracker at this point of time. Finally, the study shows that subjects typing under the $\mathrm{CW}$ light demonstrated the highest speed. It can be concluded that there is no absolute 'perfect light's colour temperature 'for office workers. However, $\mathrm{CW}$ and DL light were found to be more beneficial to office worker than the WW light.

As for recommendation, this study can be conducted in a longer period of time so that changes with visual and non-visual performance of subject could be assessed in details. Moreover, this study should be conducted by using larger sample size as it will provide results that can be generalized. 


\section{Acknowledgements}

This research was collaboration between Department of Environmental and Occupational Health, Universiti Putra Malaysia and Quality and Reliability Engineering Department, MIMOS Berhad.

\section{References}

[1] Boyce, P. R., Veitch, J. A., Newsham, G. R., Myer, M., \& Hunter, C. (2003). Lighting quality and office work: A field simulation study (PNNL-14506), Richland, WA: Pacific Northwest National Laboratory. Retrieved on October 11, 2011, from http://www.nrccnrc.gc.ca/obj/irc/doc/pubs/b3214.1/B3214.1.pdf

[2] Veitch, J. A. \& Newsham, G. R. (1998). Determinants of lighting quality 1: State of science. Journal of the Illuminating Engineering Society 27(1), 92-106

[3] Halonen, L., Tetri, E., \& Bhusal, P. (2010). Chapter 3: Lighting Quality. In L. Halonen, E. Tetri, \& P. Bhusal (Eds.), Guidebook on energy efficient electric lighting for buildings (pp 41-56). Finland: Aalto University School of Science and Technology.

[4] Górnicka, G. (2008). Lighting at work: Environmental study of direct effects of lighting level and spectrum on psychophysiological variables. Eindhoven: Technische Universiteit Eindhoven.

[5] Bullough, J., Figueiro, M., and Marilyn R. P. (2000) IESNA Lighting Handbook. 9th ed. Illuminating Engineering Society of North America, 2000. p. 10-13.

[6] Linhart, F. \&Scartezzini, J. L. (2011). Evening office lighting - visual comfort vs. energy efficiency vs. performance? Building and Environment, 46, 981-989.

[7] Eklund, N., and Boyce, P. 1996. The development of a reliable, valid and simple office lighting survey. Journal of the Illuminating Engineering Society.

[8] Goh, K.N., Chen, Y.Y, Lai, F.W., Daud,S.C., Sivaji, A., Soo, S.T. A Comparison of Multiple Usability Testing Methods to Evaluate and Analyze An e-Commerce Website: A Malaysian Case Study on An Online Gift Shop, Tenth International Conference on Information Technology - New Generations, 2013 in press

[9] Robert, G., and Garza, A. (2002). Task Lighting for the Elderly. Journal of the Illuminating Engineering Society, Volume 31, p.20-32.

[10] Park, B. C., Chang, J. H., Kim, Y. S., Jeong, J. W. \& Choi, A. S. (2010). A Study on the Subjective Response for Corrected Colour Temperature Conditions in a Specific Space. Indoor Built Environ 19(6), 623-637

[11] Cajochen. C., Zeitzer. J.M., Czeisler. C.A., and Dijk. D.J. 2000. Dose-response relationship for light intensity and ocular and electroencephalographic correlates of human alertness. Behav Brain Res 2000;115:75-83.

[12] Miller, H. (2007). Bright Idea: Personal Control for Office Lighting. Research Summary Retrieved on 5 August, 2012, from www.hermanmiller.com/research/researchsummaries/bright-idea-personal-control-for-office-lighting.html

[13] van Bommel, W. J. M. \& van den Beld, G. J. (2004). Lighting for work: a review of visual and biological effects. Lighting Res. Technology 36(4), 255-269

[14] Rautkylä, E., Halonen, L., Lehtovaara, J. (2008). Effects of artificial light spectrum on alertness: Vava - field study. Report 54, Lighting unit, Helsinki University of Technology.

[15] Lehrl, S., Gerstmeyer, K., Jacob, J.H., Frieling, H., Henkel, A.W., Meyrer, R., Wiltfang, J., Kornhuber, J., \&Bleich, S. (2007). Blue light improves cognitive performance. J. Neural Transm. 114, 457-460

[16] Sia, C. C., and Shamsul, B.M.T. (2011). Effects of Light's Colour Temperature on Visual Comfort Level, Task Performance and Alertness Among UPM'S Students. 2nd RECOUP International Conference on Ergonomics. 